Discrete Comput Geom 35:311-328 (2006)

DOI: $10.1007 / \mathrm{s} 00454-005-1221-\mathrm{z}$

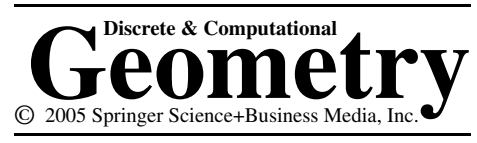

\title{
The Angle Defect for Odd-Dimensional Simplicial Manifolds
}

Ethan D. Bloch

Bard College, Annandale-on-Hudson, NY 12504, USA

bloch@bard.edu

\begin{abstract}
In a 1967 paper, Banchoff stated that a certain type of polyhedral curvature, that applies to all finite polyhedra, was zero at all vertices of an odd-dimensional polyhedral manifold; one then obtains an elementary proof that odd-dimensional manifolds have zero Euler characteristic. In a previous paper, the author defined a different approach to curvature for arbitrary simplicial complexes, based upon a direct generalization of the angle defect. The generalized angle defect is not zero at the simplices of every odd-dimensional manifold. In this paper we use a sequence based upon the Bernoulli numbers to define a variant of the angle defect for finite simplicial complexes that still satisfies a Gauss-Bonnet-type theorem, but is also zero at any simplex of an odd-dimensional simplicial complex $K$ (of dimension at least 3), such that $\chi\left(\operatorname{link}\left(\eta^{i}, K\right)\right)=2$ for all $i$-simplices $\eta^{i}$ of $K$, where $i$ is an even integer such that $0 \leq i \leq n-1$. As a corollary, an elementary proof is given that any such simplicial complex has Euler characteristic zero.
\end{abstract}

\section{Introduction}

For a triangulated polyhedral surface $M^{2}$, the usual notion of curvature at a vertex $v$ is the classical angle defect $d_{v}=2 \pi-\sum \alpha_{i}$, where the $\alpha_{i}$ are the angles of the triangles containing $v$. This curvature function, which goes back at least as far as Descartes (see $[\mathrm{F}]$ ), satisfies all the standard properties one would expect a curvature function on polyhedra to satisfy. For example, the angle defect is invariant under local polyhedral isometries (that is, functions that preserve the lengths of edges); it is locally defined; it is zero at a vertex that has a flat star; it is invariant under subdivision; and it satisfies the polyhedral Gauss-Bonnet theorem, which says $\sum_{v} d_{v}=2 \pi \chi\left(M^{2}\right)$, where the summation is over all the vertices of $M^{2}$, and $\chi\left(M^{2}\right)$ is the Euler characteristic of $M^{2}$.

There are two approaches to generalizing the classical angle defect to arbitrary (finite) polyhedra in all dimensions. One method, which we refer to as standard curvature, has been studied extensively from a differential geometric point of view. See, among others, $[\mathrm{Ba} 1],[\mathrm{W}],[\mathrm{Bu}],[\mathrm{C}],[\mathrm{CMS}]$, and $[\mathrm{Z}]$. This approach to generalizing the angle defect, 
which is based on exterior angles, is simple to define, and its convergence properties have been well studied. In standard curvature all the curvature is concentrated at the vertices, as is the case for the classical angle defect of polyhedral surfaces. Another approach, called simply the angle defect (also known as the angle deficiency), has been studied in the case of convex polytopes by a number of combinatorialists, for example, [Sh2] and [Gr2]; more generally, for the wider study of angle sums in convex polytopes and beyond, see, for example, [Gr1, Chapter 14], [Sh1], [PS], [Mc1], and [Ch1]. In [GS] a GaussBonnet-type theorem (also referred to as Descartes' theorem) is proved for the angle defect in polytopes with underlying spaces that are manifolds. In contrast to standard curvature, which is concentrated at vertices, the angle defect for convex polytopes is found at each simplex of codimension at least 2 (it can be defined for all simplices, but the angle defect at a codimension 0 or 1 simplex will always be zero). One treatment of curvature of polyhedra that has some of the advantages of all the approaches cited above is in [Ba3], which uses curvature functions based on critical points (similarly to [Ba1]), but this time using projection maps $\mathbb{R}^{n} \rightarrow \mathbb{R}^{m}$, which leads to curvature functions that both generalize standard curvature and are related to the Grassmann angles of [Gr2]; in this approach the curvature is located at all simplices, not just at vertices, and an angle defect type formula for curvature is obtained using projection maps $\mathbb{R}^{n} \rightarrow \mathbb{R}^{n-1}$.

In [B11] we extended the notion of angle defect to arbitrary simplicial complexes, not just manifolds, by using a simple toplogical decomposition of each simplicial complex. In order to compare our approach with standard curvature, we originally concentrated our curvature at the vertices, and called it stratified curvature, see Section 3 of [B11] for details. In Section 4 of [B11] we took the variant approach most directly comparable to the combinatorial authors listed above, in that we kept the pure angle defects defined for each simplex of codimension at least 2 without artificially concentrating them at the vertices. In Section 4 of [B11] we referred to this approach by the unfortunate name "modified stratified curvature," which really misses the point that in this approach we are really still working with a pure angle defect. Hence, in the present paper, we use the better name "generalized angle defect"; we used this better name in [B12].

A detailed comparison of standard curvature with both stratified curvature and the generalized angle defect may be found in Section 4 of [B11]. We mention here, however, that all these approaches satisfy some of the basic properties that one would expect of curvature, such as being locally defined, invariant under local isometries, and satisfying a Gauss-Bonnet-type theorem (though the Gauss-Bonnet theorem for stratified curvature and the generalized angle defect uses a modified Euler characteristic rather than the standard Euler characteristic, as discussed in Section 2 of [B11]). In [B12] we showed that the generalized angle defect has a Morse theoretic interpretation very similar to (though not quite as simple as) the Morse theoretic approach to standard curvature found in [Ba1]-[Ba3]. Hence, on most counts, it is fair to say that the generalized angle defect behaves as nicely as standard curvature.

There is one place, however, where the generalized angle defect falls short of standard curvature. In Section 5 of [Ba1], Banchoff stated that for an odd-dimensional polyhedral manifold, the standard curvature is zero at every vertex. (The proof of the main step of this theorem is not given in the paper; according to a private communication, the author knew that the result would be superseded by [Ba3], and hence omitted the details. The proof is not difficult, and it proceeds by induction on the dimension of the 
manifold.) It then follows from the Gauss-Bonnet theorem for standard curvature that every odd-dimensional polyhedral manifold has Euler characteristic zero (a well-known fact, but Banchoff's method yields a completely elementary proof, without using algebraic topology). By contrast, as we will see in Section 3, there are odd-dimensional compact simplicial manifolds for which neither stratified curvature nor the generalized angle defect is identically zero. The purpose of the present paper is to define a variant on the generalized angle defect called ascending stratified curvature where ascending stratified curvature satisifies most of the nice properties of the generalized angle defect, and yet also has the additional property that it is identically zero for any odd-dimensional simplicial complex $K$ (of dimension at least 3) such that $\chi\left(\operatorname{link}\left(\eta^{i}, K\right)\right)=2$ for all $i$ simplices $\eta^{i}$ of $K$, where $i$ is an even integer such that $0 \leq i \leq n-1$.

As a corollary to our approach, we deduce that if $K$ is an odd-dimensional simplicial complex (of dimension at least 3) such that $\chi\left(\operatorname{link}\left(\eta^{i}, K\right)\right)=2$ for all $i$-simplices $\eta^{i}$ of $K$, where $i$ is an even integer such that $0 \leq i \leq n-1$, then $K$ has Euler characteristic zero (see Corollary 3.7 below). This result has interesting antecedents, involving a theorem that was independently re-discovered (at least) twice. As mentioned above, in [Ba1] Banchoff gave an elementary proof that any odd-dimensional polyhedral manifold has Euler characteristic zero. In his later paper [Ba3], Banchoff gave a very efficient proof of the fact that any odd-dimensional Euler space has Euler characteristic zero, where an $n$-dimensional Euler space is an $n$-dimensional simplicial complex $K$ such that $\chi\left(\operatorname{link}\left(\eta^{i}, K\right)\right)=\chi\left(S^{n-i-1}\right)=1+(-1)^{n-i-1}$ for all $i$-simplices $\eta^{i}$ of $K$, where $i$ is any integer such that $0 \leq i \leq n$. (In [Ba3] a slightly different formulation for the definition of Euler space is given, though these two definitions are equivalent in the case of simplicial complexes.) Given that any manifold is an Euler space, but not vice versa, the result in [Ba3] is stronger than the result in [Ba1]. This same result about the Euler characteristic of odd-dimensional Euler spaces was independently re-proved in [CY2, Corollary 2.5] and [MR], neither of which refers to [Ba3], nor to each other. The paper [CY2] proves the result about odd-dimensional Euler spaces as a corollary to some results relating to the Dehn-Sommerville equations, whereas [MR] is a short note dedicated solely to a proof of this theorem. In all three of [Ba3], [CY2], and [MR] the proofs appear to use the full definition of Euler space, and they cannot drop the requirement on the links of odd-dimensional simplices as we do in Corollary 3.7. (In a private communication, the second-named author of [MR] said that they had in fact used their method to prove the equivalent of our Corollary 3.7 in dimensions 3 and 5, but were not able to prove it in general using their methods.) Euler spaces, which are also called Eulerian manifolds and Euler complexes, arise in a number of contexts; they were introduced in $[\mathrm{Kl}]$ in relation to the study of linear equations of $f$-vectors (see [CY1] and [CY2] for further discussion of this topic), and they also arise in the study of combinatorial characteristic classes, starting with $[\mathrm{Su}]$ which stressed the role of mod 2 Euler spaces (see also [HT], among many others, for more on this topic).

The outline of this paper is as follows. For the sake of completeness, we start off in Section 2 with a very brief review of the needed definitions and theorems from [B11], leaving all the details to that paper. We give our new definitions and statements of results in Section 3, and then give proofs in Section 4.

Throughout this paper we restrict our attention to simplicial complexes, rather than more general polyhedra. We make the following assumptions, which we use throughout 
this paper without stating: All simplicial complexes are finite, and are embedded in Euclidean space (which we will not name when it is not necessary); all manifolds are compact and without boundary. We assume that all simplicial complexes have dimension at least 2 , both because angle sums are trivial in dimension 1 , and to avoid special cases.

\section{Review of the Generalized Angle Defect}

We give here a very brief review of those definitions and statements of results from [B11] that we will be using, leaving proofs and examples to the original paper.

Throughout this section, let $K$ be an $n$-dimensional simplicial complex. Whereas in [B11] we allow for a certain class of non-embedded simplicial complexes, here for convenience we look only at actual simplicial complexes in Euclidean space. If $\eta$ and $\sigma$ are simplices in $K$, we write $\eta \prec \sigma$ to indicate that $\eta$ is a face of $\sigma$. As usual, we let $|K|$ denote the underlying space of $K$.

For convenience (though not necessity), we adopt the convention that all angles are normalized so that the volume of the unit $(n-1)$-sphere in $(n-1)$-measure is one in all dimensions. For any $n$-simplex $\sigma^{n}$ in Euclidean space, and any $i$-face $\eta^{i}$ of $\sigma^{n}$, let $\alpha\left(\eta^{i}, \sigma^{n}\right)$ denote the solid angle in $\sigma^{n}$ along $\eta^{i}$, where by normalization such an angle is always a number in the interval $[0,1]$.

Remark 2.1. Let $\sigma^{n}$ be an $n$-simplex. Observe that $\alpha\left(\eta^{n-1}, \sigma^{n}\right)=\frac{1}{2}$ for any $(n-1)$ face $\eta^{n-1}$ of $\sigma^{n}$, and that $\alpha\left(\sigma^{n}, \sigma^{n}\right)=1$.

Definition. For each non-negative integer $i$, let $T_{i}$ denote the open cone on $i$ points; alternately, $T_{i}$ is the space obtained by gluing together $i$ copies of the half open interval $[0,1)$ at the point $\{0\}$ in each. We take $T_{0}$ to be a single point. See Fig. 1 . Let $P_{n, i}$ denote the space $P_{n, i}=T_{i} \times \mathbb{R}^{n-1}$. If $*$ denotes the cone point of $T_{i}$, we call $\{*\} \times \mathbb{R}^{n-1} \subseteq P_{n, i}$ the apex set of $T_{i}$. See Fig. 2.

Observe that $P_{n, i}$ is not homeomorphic to $P_{n, j}$ when $i \neq j$.

We now need to think of simplices as open (and hence disjoint).

Definition. Let $K$ be an $n$-dimensional simplicial complex. For each non-negative integer $r$ such that $r \neq 2$, we define the subset $C_{r}^{n}(K)$ of $|K|$ by

$$
\begin{aligned}
& C_{r}^{n}(K)=\left\{x \in|K| \mid x \text { has neighborhood homeomorphic to } P_{n, r},\right. \\
&\text { where the homeomorphism takes } \left.x \text { to the apex set of } P_{n, r}\right\} .
\end{aligned}
$$

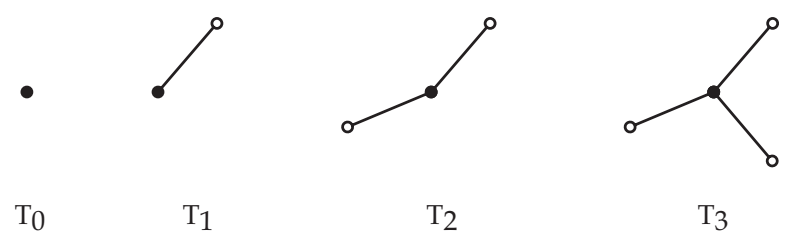

Fig. 1 


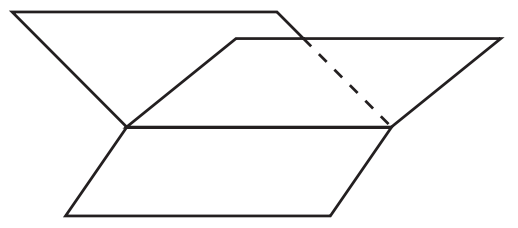

Fig. 2

Define

$$
C_{2}^{n}(K)=|K|-\bigcup_{r \neq 2} C_{r}^{n}(K)
$$

Remark 2.2. (1) Let $K$ be an $n$-dimensional simplicial complex. The sets $C_{r}^{n}(K)$ are well defined, since each $x \in|K|$ can have a neighborhood homeomorphic to $P_{n, r}$ (where the homeomorphism takes $x$ to the apex set of $P_{n, r}$ ) for at most one number $r$. Moreover, the sets $C_{r}^{n}(K)$ are well defined up to homeomorphism of $|K|$.

(2) Because $K$ is a finite simplicial complex, there is some positive integer $P$ such that $C_{r}^{n}(K)=\emptyset$ for all $r>P$.

(3) The sets $C_{r}^{n}(K)$ are disjoint, and cover $|K|$. For each $r \neq 2$, the set $C_{r}^{n}(K)$ is an $(n-1)$-manifold. Moreover, each set $C_{r}^{n}(K)$ is the union of (open) simplices of $K$, since all points in any simplex of $K$ have homeomorphic neighborhoods in $|K|$ (if the neighborhoods are taken small enough). If $\sigma \in K$, then $\sigma \subseteq C_{r}^{n}(K)$ for some unique integer $r$.

Definition. Let $K$ be an $n$-dimensional simplicial complex. For each simplex $\sigma \in K$, we define the number $T_{n}(\sigma)$ by $T_{n}(\sigma)=r / 2$, where $\sigma \in C_{r}^{n}(K)$ for some unique integer $r$.

Remark 2.3. Let $K$ be an $n$-dimensional simplicial complex. If $\eta^{n-1}$ is an $(n-1)$ simplex of $K$, then $T_{n}\left(\eta^{n-1}\right)$ equals one half the number of $n$-simplices of $K$ that have $\eta^{n-1}$ as a face. If $\sigma^{n}$ is an $n$-simplex of $K$, then $T_{n}\left(\sigma^{n}\right)=1$.

The following definition was originally given in Section 4 of [B11], though here we use the better name given below (and also used in [B12], as discussed in Section 1). In contrast to standard curvature, which in all dimensions is concentrated at the vertices (see [Ba1] and [CMS] for example), our approach has curvature at all simplices (though the non-zero curvature is always at simplices of codimension at least 2), similarly to the combinatorial approach (see [Sh2] and [Gr2] for example), as well as the geometric approach of [Ba3].

Definition. Let $K$ be an $n$-dimensional simplicial complex, and let $\eta^{i}$ be an $i$-simplex of $K$, where $0 \leq i \leq n$. The generalized angle defect at $\eta^{i}$ is the number $\tilde{D}_{n}\left(\eta^{i}\right)$ defined by

$$
\tilde{D}_{n}\left(\eta^{i}\right)=T_{n}\left(\eta^{i}\right)-\sum_{\sigma^{n}>\eta^{i}} \alpha\left(\eta^{i}, \sigma^{n}\right),
$$

where the summation is over all $n$-simplices $\sigma^{n}$ which have $\eta^{i}$ as a face. 
Remark 2.4. Using (1), together with Remarks 2.1 and 2.3 , it is seen that $\tilde{D}_{n}\left(\eta^{i}\right)=0$ if $i=n-1$ or $i=n$.

In Section 3 of [B11] we gave the following variant definition of curvature of simplicial complexes; this definition concentrates all curvature at the vertices, which is somewhat unnatural, but was used to facilitate a comparison with the definition of curvature found in [Ba1].

Definition. Let $K$ be an $n$-dimensional simplicial complex, and let $v$ be a vertex of $K$. The stratified curvature at $v$ is the number $\tilde{D}_{n}\left(\eta^{i}\right)$ defined by

$$
D_{n}(v, K)=\sum_{i=0}^{n-2} \frac{(-1)^{i}}{i+1} \sum_{\eta^{i} \ni v} \tilde{D}_{n}\left(\eta^{i}\right)
$$

In [B11] we proved Gauss-Bonnet-type theorems for both generalized angle defect and stratified curvature, though rather than using the standard Euler characteristic, we used the following variant of the Euler characteristic. We use this new characteristic in the present paper as well.

Definition. Let $K$ be an $n$-dimensional simplicial complex in $\mathbb{R}^{m}$. The number $\chi^{s}(K)$ is defined by

$$
\chi^{s}(K)=\sum_{\eta \in K} T_{n}(\eta)(-1)^{\operatorname{dim} \eta}
$$

The above definition is a particular case of the weighted Euler characteristics discussed in [Ch2] and [CY1]. In the notation of those two papers, the symbol $\chi^{s}(K)$ would be written $\chi(K, T)$, but we do not need this latter notation, because we never use a different weight on the simplices.

Remark 2.5. As discussed in Section 2 of [B11], we know that $\chi^{s}(K)$ is a homeomorphism invariant of $|K|$, though not a homotopy-type invariant.

\section{Statement of Results}

We start by showing that there are odd-dimensional compact simplicial manifolds for which neither stratified curvature nor the generalized angle defect is identically zero. We make use of convex polytopes in our discussion. For general information on convex polytopes, see [Gr1]. As usual, if $Q$ is a simplicial complex, we let $f_{i}(Q)$ denote the number of $i$-simplices of $Q$.

To see that the generalized angle defect is not identically zero for odd-dimensional simplicial manifolds is simple. Let $K$ be the boundary of any $n$-dimensional convex polytope in $\mathbb{R}^{n}$ with $n$ an even integer such that $n \geq 4$. Hence $K$ is an $(n-1)$-dimensional simplicial sphere. Let $\eta^{i}$ be an $i$-simplex of $K$ with $0 \leq i \leq n-2$. Then by Theorem (3) of [Sh2], we know that $\tilde{D}_{7}\left(\eta^{i}\right)>0$. Hence the generalized angle defect is certainly not zero for all simplices of all odd-dimensional combinatorial manifolds. 
We now turn to stratified curvature, where we need a slightly more complicated counterexample. (The simplest counterexample we found is seven-dimensional; it can be verified that there cannot be a three-dimensional counterexample, but the author does not know whether a five-dimensional counterexample could exist, though one cannot be constructed by our method.)

Example 3.1. Take a triangle in the plane, and let $Q$ be the cone of the cone of the suspension of the triangle. Then $Q$ is a five-dimensional convex polytope in $\mathbb{R}^{5}$. Let $L$ be the boundary of $Q$, so that $L$ is a four-dimensional simplicial sphere. It is straightforward to see that $f_{0}(L)=7, f_{1}(L)=20, f_{2}(L)=29, f_{3}(L)=22$, and $f_{4}(L)=8$. Triangulate $Q$ by putting a vertex $v$ in the interior of $Q$, and then joining $v$ to $L$.

Next, let $M$ be the boundary of an eight-dimensional simplicial convex polytope in $\mathbb{R}^{8}$, so that $M$ is a seven-dimensional simplicial sphere; it does not matter what simplicial convex polytope is used. Let $\eta^{5}$ be some 5 -simplex in $M$. As mentioned above, we know that $\tilde{D}_{7}\left(\eta^{5}\right)>0$. We subdivide $M$ as follows. First, put a copy of the triangulation of $Q$ in the interior of $\eta^{5}$. Then add further simplices to $M$, without subdividing $Q$, so that we obtain a simplicial complex, denoted $M^{\prime}$.

Suppose that $\alpha^{i}$ is an $i$-simplex of $M^{\prime}$. Then $\alpha^{i}$ is contained in a unique $r$-simplex $\zeta^{r}$ of $M$, where $i \leq r \leq 7$ (recall that we are thinking of simplices as open, and hence disjoint). It is seen that $T_{7}\left(\alpha^{i}\right)=T_{7}\left(\zeta^{r}\right)$ and $\tilde{D}_{7}\left(\alpha^{i}\right)=\tilde{D}_{7}\left(\zeta^{r}\right)$, where the left-hand side of each of these equations is with respect to $M^{\prime}$, and the right-hand side is with respect to $M$.

Let $\omega^{i}$ be a simplex in $M^{\prime}$ that has $v$ as a vertex. If $\omega^{i}$ is contained in $\eta^{5}$ (and hence it is contained in $Q$ ), then $\tilde{D}_{7}\left(\omega^{i}\right)=\tilde{D}_{7}\left(\eta^{5}\right)$. If $\omega^{i}$ is not contained in $\eta^{5}$, then it is contained in one of the six- or seven-dimensional simplices of $M$ that have $\eta^{5}$ as a face. By Remark 2.4, it follows that $\tilde{D}_{7}\left(\omega^{i}\right)=0$. Now, using (2), and keeping track of the simplices of $Q$ that contain $v$ by looking at $\operatorname{link}(v, Q)=L$, we see that

$$
\begin{aligned}
D_{7}\left(v, M^{\prime}\right)= & \tilde{D}_{7}\left(\eta^{5}\right)-\frac{1}{2} f_{0}(L) \tilde{D}_{7}\left(\eta^{5}\right)+\frac{1}{3} f_{1}(L) \tilde{D}_{7}\left(\eta^{5}\right)-\frac{1}{4} f_{2}(L) \tilde{D}_{7}\left(\eta^{5}\right) \\
& +\frac{1}{5} f_{3}(L) \tilde{D}_{7}\left(\eta^{5}\right)-\frac{1}{6} f_{4}(L) \tilde{D}_{7}\left(\eta^{5}\right) \\
= & -\frac{1}{60} \tilde{D}_{7}\left(\eta^{5}\right) \neq 0 .
\end{aligned}
$$

Hence we have an example of an odd-dimensional simplicial manifold with non-zero standard curvature at one of its vertices. (We note that this example is a combinatorial manifold, using the fact that all simplicial convex polytopes, and all subdivisions of simplicial convex polytopes, are combinatorial manifolds; this follows from standard results about combinatorial manifolds, as found in Section $\mathrm{I} .5 \mathrm{of}[\mathrm{H}]$, for example).

Our goal is to reformulate the notion of curvature for simplicial complexes in terms of the generalized angle defect, with the aim of remedying the above problem; that is, we want a notion of curvature based on the generalized angle defect, but which is identically zero at every simplex of odd-dimensional simplicial manifolds, and also satisfies the nice properties of the generalized angle defect, such as a Gauss-Bonnet-type theorem. Certainly, the most natural way to look at the generalized angle defect is simply to use $\tilde{D}_{n}\left(\tau^{p}\right)$ as defined for each simplex $\tau^{p}$ in a simplicial complex. In stratified curvature, 
we concentrated the $\tilde{D}_{n}\left(\tau^{p}\right)$ at the vertices via (2), to obtain $D_{n}(v, K)$. Concentrating the curvature of a simplicial complex at the vertices is not useful for our present purposes, but it raises the question of whether there is some other way of redistributing the generalized angle defects in such a way that the other desired properties of simplicial curvature still hold (for example, locally defined, invariant under local isometries, and a GaussBonnet-type theorem), and yet the property that the curvature is zero at all simplices in odd-dimensional simplicial manifolds also holds.

What does it mean for curvature to be locally defined in a simplicial complex? In the smooth case, for comparison, the fact that curvature is locally defined means that it can be calculated on an arbitrarily small neighborhood of each point. In the simplicial case, a number associated to each simplex is local if it can be defined using only the star of each simplex. In particular, let $K$ be an $n$-dimensional simplicial complex, and let $\tau^{p}$ be a $p$-simplex of $K$. Anything that can be computed in $\operatorname{star}\left(\tau^{p}, K\right)$ can be considered to be locally defined at $\tau^{p}$. For example, clearly $\tilde{D}_{n}\left(\tau^{p}\right)$ is locally defined at $\tau^{p}$. However, if $\eta^{i}$ has $\tau^{p}$ as a face (so that $\eta^{i} \in \operatorname{star}\left(\tau^{p}, K\right)$ ), then $\operatorname{star}\left(\eta^{i}, K\right)$ is contained in $\operatorname{star}\left(\tau^{p}, K\right)$, and hence $\tilde{D}_{n}\left(\eta^{i}\right)$ is also locally defined at $\tau^{p}$. Therefore, we see that any number produced out of $\tilde{D}_{n}\left(\tau^{p}\right)$ and all the $\tilde{D}_{n}\left(\eta^{i}\right)$ for $\eta^{i}$ that have $\tau^{p}$ as a face is locally defined at $\tau^{p}$. Of course, a number produced out of an arbitrarily chosen combination of generalized angle defects will not necessarily behave nicely as one would expect of a curvature function. There is, we will see, one method of combining these generalized angle defects that has the desired properties.

To define the desired curvature, we will need the following definition, which uses the Bernoulli numbers. (See, for example, Sections 6.5 and 7.6 of [GKP] for more on the Bernoulli numbers.) As usual, we let $B_{k}$ denotes the $k$ th Bernoulli number.

Definition. For each non-negative integer $n$, let $a_{n}$ be the number defined by

$$
a_{n}=\frac{4 B_{n+2}\left(2^{n+2}-1\right)}{n+2} .
$$

We refer to the sequence $a_{0}, a_{1}, a_{2}, \ldots$ as the angle defect sequence.

The first 18 numbers in the angle defect sequence are

$$
1,0,-\frac{1}{2}, 0,1,0,-\frac{17}{4}, 0,31,0,-\frac{691}{2}, 0,5461,0,-\frac{929569}{8}, 0,3202291,0 .
$$

The definition of the angle defect sequence appears in the literature, though there does not appear to be a name for this sequence, and hence we use the name we have given. The non-zero numerators of the angle defect sequence can be found in the wonderful website [Sl]. See p. 508 of $[\mathrm{Kn}]$ for an older printed reference to the angle defect sequence, and see p. 95 of [RW] for a recent appearance, in relation to numbers of simplices and angle defects of simplicial complexes.

The following lemma gives some properties of the angle defect sequence that we will use later; the proof of the lemma is given in Section 4. 


\section{Lemma 3.2.}

(1) For every odd integer $n$, we have $a_{n}=0$.

(2) The angle defect sequence satisfies the recursion relation $a_{0}=1$, and

$$
a_{n}+\sum_{i=0}^{n-1} \frac{a_{i}}{2}\left(\begin{array}{c}
n+1 \\
i+1
\end{array}\right)=1
$$

for all positive integers $n$.

We can now give the definition of our new type of curvature.

Definition. Let $a_{0}, a_{1}, a_{2}, \ldots$ be the angle defect sequence. Let $K$ be an $n$-dimensional simplicial complex, and let $\tau^{p}$ be a $p$-simplex of $K$. The ascending stratified curvature at $\tau^{p}$ is the number $D_{n}^{a}\left(\tau^{p}\right)$ defined by

$$
D_{n}^{a}\left(\tau^{p}\right)=a_{p} \tilde{D}_{n}\left(\tau^{p}\right)+\frac{a_{p}}{2} \sum_{i=p+1}^{n}(-1)^{i-p} \sum_{\eta^{i} \succ \tau^{p}} \tilde{D}_{n}\left(\eta^{i}\right) .
$$

Remark 3.3. (1) Using Remark 2.4, it is seen that $D_{n}^{a}\left(\tau^{p}\right)=0$ if $p=n-1$ or $p=n$. Moreover, when $0 \leq p \leq n-2$, we have

$$
D_{n}^{a}\left(\tau^{p}\right)=a_{p} \tilde{D}_{n}\left(\tau^{p}\right)+\frac{a_{p}}{2} \sum_{i=p+1}^{n-2}(-1)^{i-p} \sum_{\eta^{i} \succ \tau^{p}} \tilde{D}_{n}\left(\eta^{i}\right) .
$$

(2) Because $a_{p}=0$ for all odd $p$ (by Lemma 3.2(1)), it follows that $D_{n}^{a}\left(\tau^{p}\right)=0$ for all odd $p$. Hence, the ascending stratified curvature is concentrated at the evendimensional simplices. (We could have defined ascending stratified curvature only for even-dimensional simplices; however, it is more convenient to define the curvature are we did, including the odd-dimensional simplices.)

It is straightforward to check that ascending stratified curvature reduces to the classical angle defect when $K$ is a simplicial surface.

Our goal is to show that ascending stratified curvature is nicely behaved. Clearly it is locally defined, and invariant under local isometries. Our three main theorems, which we now state, say that ascending stratified curvature satisfies a Gauss-Bonnettype theorem (using $\chi^{s}(K)$ ), that it is identically zero for odd-dimensional compact simplicial manifolds, and that it is somewhat invariant under subdivision (to be clarified below). The proofs of these results are given in Section 4. Our first theorem is the following.

Theorem 3.4. $\quad$ Let $K$ be an $n$-dimensional simplicial complex, where $n \geq 2$. Then

$$
\sum_{\tau^{p} \in K}(-1)^{p} D_{n}^{a}\left(\tau^{p}\right)=\chi^{s}(K) .
$$


The following theorem gives a general condition that guarantees that ascending stratified curvature will be identically zero in the odd-dimensional case.

Theorem 3.5. Let $K$ be an $n$-dimensional simplicial complex, where $n$ is an odd integer such that $n \geq 3$. Suppose that $\chi\left(\operatorname{link}\left(\eta^{i}, K\right)\right)=2$ for all $i$-simplices $\eta^{i}$ of $K$, where $i$ is an even integer such that $0 \leq i \leq n-1$. Then $D_{n}^{a}(\tau)=0$ for every simplex $\tau$ of $K$.

The following corollary is deduced immediately from Theorem 3.5, using the fact that the links of simplices in homology manifolds have the appropriate homology groups (and hence Euler characteristic); see Theorem 63.2 of [Mu] for more details. Recall that we are assuming that all simplicial complexes are finite, and all manifolds are compact and without boundary.

Corollary 3.6. Let $K$ be a simplicial homology $n$-manifold, where $n$ is an odd integer such that $n \geq 3$. Then $D_{n}^{a}(\tau)=0$ for every simplex $\tau$ of $K$.

The following result is another simple corollary to Theorem 3.5.

Corollary 3.7. Let $K$ be an $n$-dimensional simplicial complex, where $n$ is an odd integer such that $n \geq 3$. Suppose that $\chi\left(\operatorname{link}\left(\eta^{i}, K\right)\right)=2$ for all $i$-simplices $\eta^{i}$ of $K$, where $i$ is an even integer such that $0 \leq i \leq n-1$. Then $\chi(K)=0$.

Proof. Combining Theorems 3.5 and 3.4, it follows that $\chi^{s}(K)=0$. The condition on the links of even-dimensional simplices implies that every $(n-1)$-simplex of $K$ is the face of precisely two $n$-simplices. It follows from Remark 2.3 that $T_{n}\left(\tau^{p}\right)=1$ for all $p$-simplices $\tau^{p}$ of $K$, where $p=n-1$ or $p=n$. Moreover, by using Lemma 4.1(i), stated and proved below, it follows that $T_{n}\left(\tau^{p}\right)=1$ for all $p$-simplices $\tau^{p}$ of $K$, where $0 \leq p \leq n-2$. It now follows from (3) that $\chi^{s}(K)=\chi(K)$.

Corollary 3.7 immediately implies the following standard result, which is usually proved by algebraic topology, though our method yields a completely elementary proof of this fact.

Corollary 3.8. Let $K$ be a triangulated homologyn-manifold, where $n$ is an odd integer such that $n \geq 3$. Then $\chi(K)=0$.

The one remaining property of ascending stratified curvature we wish to examine is invariance under subdivision. Let $K$ be an $n$-dimensional simplicial complex, where $n \geq 2$, and let $L$ be a subdivision of $K$. Suppose that $\tau^{s}$ is an $s$-simplex of $L$. Then $\tau^{s}$ is contained in a unique $s$-simplex $\zeta^{p}$ of $K$, where $s \leq p \leq n$ (recall that we are thinking of simplices as open, and hence disjoint). To say that ascending stratified curvature is invariant under subdivision would, in its nicest form, mean precisely that $D_{n}^{a}\left(\tau^{p}\right)=D_{n}^{a}\left(\zeta^{r}\right)$, where the left-hand side of this equation is with respect to $L$, and the right-hand side is with respect to $K$. Because of the use of the angle defect sequence in the definition of ascending stratified curvature, we do not quite have this simple equation, but we have a slightly modified version of it instead, as stated in the following theorem. 
Theorem 3.9. $\quad$ Let $K$ be an $n$-dimensional simplicial complex, where $n \geq 2$, and let $L$ be a subdivision of $K$. Let $\tau^{s}$ be an $s$-simplex of $L$ that is contained in a p-simplex $\zeta^{p}$ of $K$. Then

$$
a_{p} D_{n}^{a}\left(\tau^{s}\right)=a_{s} D_{n}^{a}\left(\zeta^{p}\right)
$$

When $s=p$, then

$$
D_{n}^{a}\left(\tau^{p}\right)=D_{n}^{a}\left(\zeta^{p}\right)
$$

For comparison, we note that both standard curvature and the generalized angle defect are invariant under subdivision (as stated in Section 4 of [Bl1]), with no need for the modification introduced in (9).

Finally, we note that the angle defect sequence $a_{0}, a_{1}, a_{2}, \ldots$ used in the definition of ascending stratified curvature is crucial. Although in principle one could define analogs of ascending stratified curvature by using different sequences $a_{0}, a_{1}, a_{2}, \ldots$ in (6), such curvature functions would not satisfy all our properties. A look at the proofs (given below) of Theorems 3.4, 3.5, and 3.9 shows that whereas Theorem 3.9 would still hold with any sequence $a_{0}, a_{1}, a_{2}, \ldots$ in (6), Theorem 3.4 holds only if the sequence satisfies the recursive definition of the angle defect sequence given in Lemma 3.2(2), and Theorem 3.5 holds because $a_{n}=0$ for all odd $n$ (proved in Lemma 3.2(1)). The author finds it somewhat remarkable that there is a sequence that simultaneously satisfies both these needed properties.

The appearance of the Bernoulli numbers in connection with angle sums and the angle defect might appear surprising, but in fact such connections can be found in the literature, for example, $[\mathrm{P}],[\mathrm{Gu}]$, and especially $[\mathrm{RW}]$. This latter paper makes use of the sequence we called the angle defect sequence in relation to angle sums and angle defects in even-dimensional manifolds, and, in particular, (3.4) of [RW] gives a result similar in spirit to our Theorem 3.4, though only for even-dimensional manifolds. However, the method of $[\mathrm{RW}]$ does not yield a curvature function that satisfies the analog of our Theorem 3.5. The Bernoulli numbers also appear elsewhere in topology, as in Appendix B of [MS].

\section{Proofs}

We start with a proof of Lemma 3.2.

Proof of Lemma 3.2. (1) This follows immediately from the fact that $B_{n}=0$ for all odd integers $n$ such that $n \geq 3$ (see Section 6.5 of [GKP]).

(2) The fact that $a_{0}=1$ follows from the fact that $B_{2}=\frac{1}{6}$. To verify (5), we will need the following three formulas involving the Bernoulli numbers and the Bernoulli polynomials (denoted $B_{k}(x)$ ), the first two from Section 6.5 of [GKP], and the third from p. 805 of [AS]. For each non-negative integer $n$, we have

$$
\sum_{i=0}^{n}\left(\begin{array}{c}
n \\
i
\end{array}\right) B_{i}=B_{n}
$$




$$
\begin{aligned}
\sum_{i=0}^{n}\left(\begin{array}{l}
n \\
i
\end{array}\right) B_{i} h^{n-i} & =B_{n}(h), \\
B_{n}\left(\frac{1}{2}\right) & =-\left(1-2^{1-n}\right) B_{n} .
\end{aligned}
$$

By substituting $h=\frac{1}{2}$ into (12), and then using (13) and doing some rearranging, we obtain

$$
\sum_{i=0}^{n}\left(\begin{array}{l}
n \\
i
\end{array}\right) B_{i} 2^{i}=B_{n}\left(2-2^{n}\right) .
$$

Next, substituting the definition of the $a_{n}$, given in (4), into the left-hand side of (5), and then using (11) and (14), the fact that $B_{0}=1$ and $B_{1}=-\frac{1}{2}$, and a standard identity involving binomial coefficients, it is a straightforward computation to show that (5) holds. We leave the details to the reader.

We now prove our three theorems, starting with Theorem 3.4.

Proof of Theorem 3.4. We compute

$$
\begin{aligned}
& \sum_{\tau^{p} \in K}(-1)^{p} D_{n}^{a}\left(\tau^{p}\right) \\
& =\sum_{p=0}^{n-2} \sum_{\tau^{p} \in K}(-1)^{p} D_{n}^{a}\left(\tau^{p}\right) \\
& \text { (using the fact that } D_{n}^{a}\left(\tau^{p}\right)=0 \text { when } p=n-1 \text { or } p=n \text { ) } \\
& =\sum_{p=0}^{n-2} \sum_{\tau^{p} \in K}(-1)^{p}\left\{a_{p} \tilde{D}_{n}\left(\tau^{p}\right)+\frac{a_{p}}{2} \sum_{i=p+1}^{n-2}(-1)^{i-p} \sum_{\eta^{i} \succ \tau^{p}} \tilde{D}_{n}\left(\eta^{i}\right)\right\} \\
& =\sum_{p=0}^{n-2} \sum_{\tau^{p} \in K}(-1)^{p} a_{p} \tilde{D}_{n}\left(\tau^{p}\right)+\sum_{p=0}^{n-2} \sum_{\tau^{p} \in K} \sum_{i=p+1}^{n-2} \sum_{\eta^{i} \succ \tau^{p}}(-1)^{i} \frac{a_{p}}{2} \tilde{D}_{n}\left(\eta^{i}\right) \\
& =\sum_{p=0}^{n-2} \sum_{\tau^{p} \in K}(-1)^{p} a_{p} \tilde{D}_{n}\left(\tau^{p}\right)+\sum_{p=0}^{n-2} \sum_{i=p+1}^{n-2} \sum_{\eta^{i} \in K} \sum_{\tau^{p}<\eta^{i}}(-1)^{i} \frac{a_{p}}{2} \tilde{D}_{n}\left(\eta^{i}\right) \\
& =\sum_{p=0}^{n-2} \sum_{\tau^{p} \in K}(-1)^{p} a_{p} \tilde{D}_{n}\left(\tau^{p}\right)+\sum_{p=0}^{n-2} \sum_{i=p+1}^{n-2} \sum_{\eta^{i} \in K}(-1)^{i} \frac{a_{p}}{2}\left(\begin{array}{c}
i+1 \\
p+1
\end{array}\right) \tilde{D}_{n}\left(\eta^{i}\right) \\
& =\sum_{i=0}^{n-2} \sum_{\eta^{i} \in K}(-1)^{i} a_{i} \tilde{D}_{n}\left(\eta^{i}\right)+\sum_{i=1}^{n-2} \sum_{\eta^{i} \in K} \sum_{p=0}^{i-1}\left(\begin{array}{c}
i+1 \\
p+1
\end{array}\right)(-1)^{i} \frac{a_{p}}{2} \tilde{D}_{n}\left(\eta^{i}\right) \\
& =\sum_{\eta^{0} \in K}(-1)^{0} a_{0} \tilde{D}_{n}\left(\eta^{0}\right)+\sum_{i=1}^{n-2} \sum_{\eta^{i} \in K}(-1)^{i}\left\{a_{i}+\sum_{p=0}^{i-1}\left(\begin{array}{c}
i+1 \\
p+1
\end{array}\right) \frac{a_{p}}{2}\right\} \tilde{D}_{n}\left(\eta^{i}\right)
\end{aligned}
$$




$$
\begin{aligned}
& =\sum_{\eta^{0} \in K}(-1)^{0} 1 \cdot \tilde{D}_{n}\left(\eta^{0}\right)+\sum_{i=1}^{n-2} \sum_{\eta^{i} \in K}(-1)^{i} 1 \cdot \tilde{D}_{n}\left(\eta^{i}\right) \\
& \quad \text { (using Lem } \\
& =\sum_{i=0}^{n-2}(-1)^{i} \sum_{\eta^{i} \in K}\left\{T_{n}\left(\eta^{i}\right)-\sum_{\sigma^{n} \succ \eta^{i}} \alpha\left(\eta^{i}, \sigma^{n}\right)\right\} \quad \text { (using (1)) } \\
& =\chi^{s}(K) \quad \text { (using the computation given in p. } 387 \text { of [B11]). }
\end{aligned}
$$

We now turn to the proof of Theorem 3.5, starting with two lemmas.

Lemma 4.1. Let $K$ be an $n$-dimensional simplicial complex, where $n \geq 2$. Suppose that each $(n-1)$-simplex of $K$ is the face of precisely two $n$-simplices. Let $\tau^{p}$ be a $p$ simplex of $K$ with $0 \leq p \leq n-2$. Then

(i) $T_{n}\left(\tau^{p}\right)=1$;

(ii) $2 f_{n-p-2}\left(\operatorname{link}\left(\tau^{p}, K\right)\right)=(n-p) f_{n-p-1}\left(\operatorname{link}\left(\tau^{p}, K\right)\right)$.

Proof. (i) We use the discussion in Section 2. Recall that we are thinking of simplices as open (and hence disjoint). Using Remark 2.2(3), we know that $\tau^{p}$ is entirely contained in one of the sets $C_{r}^{n}(K)$. Suppose $r \neq 2$. By the same remark, we know that $C_{r}^{n}(K)$ is an $(n-1)$-manifold, and it is the union of simplices of $K$. Therefore it must contain at least one $(n-1)$-simplex $\eta^{n-1}$. By the definition of the set $C_{r}^{n}(K)$, it follows that $\eta^{n-1}$ must be the face of precisely $r n$-simplices of $K$. Because $r \neq 2$, we have a contradiction to the hypothesis of the lemma. Hence we conclude that $r=2$, and hence $T_{n}\left(\tau^{p}\right)=1$, using the definition of $T_{n}\left(\tau^{p}\right)$.

(ii) Let $\zeta^{n-p-1}$ be an $(n-p-1)$-simplex of $\operatorname{link}\left(\tau^{p}, K\right)$. We know that $\zeta^{n-p-1}$ has $n-p(n-p-1)$-faces, and these faces are all in $\operatorname{link}\left(\tau^{p}, K\right)$. We claim that every $(n-p-2)$-simplex of $\operatorname{link}\left(\tau^{p}, K\right)$ is the face of precisely two $(n-p-1)$-simplices of $\operatorname{link}\left(\tau^{p}, K\right)$. The desired result follows immediately from this claim. To prove the claim, let $\eta^{n-p-2}$ be an $(n-p-2)$-simplex of $\operatorname{link}\left(\tau^{p}, K\right)$. Then $\tau^{p} * \eta^{n-p-2}$ is an $(n-1)$ simplex of $K$. It is seen that for every $(n-p-1)$-simplex $\alpha^{n-p-1}$ of $\operatorname{link}\left(\tau^{p}, K\right)$ that has $\eta^{n-p-2}$ as a face, we obtain the $n$-simplex $\tau^{p} * \alpha^{n-p-1}$ of $K$ that has $\tau^{p} * \eta^{n-p-2}$ as a face. Conversely, for every $n$-simplex $\beta^{n}$ of $K$ that has $\tau^{p} * \eta^{n-p-2}$ as a face, we can write $\beta^{n}$ as $\tau^{p} * \gamma^{n-p-1}$, where $\gamma^{n-p-1}$ is an $(n-p-1)$-simplex of $\operatorname{link}\left(\tau^{p}, K\right)$ that has $\eta^{n-p-2}$ as a face. Hence the number of $(n-p-1)$-simplices of $\operatorname{link}\left(\tau^{p}, K\right)$ that have $\eta^{n-p-2}$ as a face equals the number of $n$-simplices of $K$ that have $\tau^{p} * \eta^{n-p-2}$ as a face. By hypothesis the latter number is two, and hence there are also two $(n-p-1)$ simplices of $\operatorname{link}\left(\tau^{p}, K\right)$ that have $\eta^{n-p-2}$ as a face.

Lemma 4.2. Let $n$ be an odd integer such that $n \geq 3$. Let $\sigma^{n}$ be an $n$-simplex in Euclidean space, and let $\tau^{p}$ be a p-face of $\sigma^{n}$, where $p$ is an even integer such that $0 \leq p \leq n-2$. Then

$$
\alpha\left(\tau^{p}, \sigma^{n}\right)-\frac{1}{2} \sum_{i=p+1}^{n-2}(-1)^{i+1} \sum_{\sigma^{n} \succ \eta^{i} \succ \tau^{p}} \alpha\left(\eta^{i}, \sigma^{n}\right)=\frac{1}{2}-\frac{n-p}{4},
$$

where the inner summation is over all $i$-simplices $\eta^{i}$ of $\sigma^{n}$ that have $\tau^{p}$ as a face. 
Proof. First, we show that (15) is equivalent to the equation

$$
\sum_{i=p+1}^{n}(-1)^{i-p+1} \sum_{\sigma^{n} \succ \eta^{i} \succ \tau^{p}} \alpha\left(\eta^{i}, \sigma^{n}\right)=2 \alpha\left(\tau^{p}, \sigma^{n}\right) .
$$

To see the equivalence, observe that by definition we have $\alpha\left(\sigma^{n}, \sigma^{n}\right)=1$, and that $\alpha\left(\eta^{n-1}, \sigma^{n}\right)=\frac{1}{2}$ for every $(n-1)$-face $\eta^{n-1}$ of $\sigma^{n}$. It can be verified that there are precisely $n-p(n-1)$-faces of $\sigma^{n}$ that contain $\tau^{p}$. Then, using the fact that $n$ is odd and $p$ is even, we have

$$
(-1)^{(n-1)-p+1} \sum_{\sigma^{n} \succ \eta^{n-1} \succ \tau^{p}} \alpha\left(\eta^{i}, \sigma^{n}\right)=-\frac{n-p}{2}
$$

and

$$
(-1)^{n-p+1} \sum_{\sigma^{n} \succ \eta^{n} \succ \tau^{p}} \alpha\left(\eta^{n}, \sigma^{n}\right)=1 .
$$

Substituting (17) and (18) into (16), and doing some rearranging, yields (15).

Next, we need to verify that (16) holds. This equation follows from standard results concerning angle sums in convex polytopes and convex cones, and we sketch the proof. We cite [Mc2] for some basic notation and results, though these results are found in many other sources as well; see [Gr1] for more background about convex polytopes.

Withough loss of generality, we can translate $\sigma^{n}$ so that some point in $\tau^{p}$ is taken to the origin (recall that we are thinking of simplices as open). Because all the angles under consideration are in $\sigma^{n}$, we can restrict our attention to the the affine span of $\sigma^{n}$, which we identify with $\mathbb{R}^{n}$.

Let $Q$ be the polyhedral cone with apex at the origin generated by $\sigma^{n}$. The set of apices of the cone $Q$, denoted $T$, is the affine span of $\tau^{p}$. Clearly $T$ is a $p$-dimensional linear subspace $T$ of $\mathbb{R}^{n}$ The faces of $Q$ correspond precisely to those faces of $\sigma^{n}$ that have $\tau^{p}$ as a face; for each face $\eta^{i}$ of $\sigma^{n}$ that has $\tau^{p}$ as a face, we denote its corresponding face in $Q$ by $\hat{\eta}^{i}$. Moreover, we have $\alpha\left(\hat{\eta}^{i}, Q\right)=\alpha\left(\eta^{i}, \sigma^{n}\right)$ for every appropriate $\eta^{i}$.

Next, let $P=Q \cap T^{\perp}$, where $T^{\perp}$ is the $(n-p)$-dimensional linear subspace of $\mathbb{R}^{n}$ that is perpendicular to $T$. Then $P$ is an $(n-p)$-dimensional polyhedral cone, with one apex, namely the origin. Moreover, it is seen that $Q=P \times T^{\perp}$. The faces of $P$ correspond to the faces of $Q$; more precisely, for each $i$-face $\hat{\eta}^{i}$ of $Q$, there is a corresponding $(i-p)$ face $\bar{\eta}^{i}$ of $P$, where $\bar{\eta}^{i}=\hat{\eta}^{i} \cap T^{\perp}$ and $\hat{\eta}^{i}=\bar{\eta}^{i} \times T^{\perp}$. Then, using Lemma 2 from [Mc1], and the fact that our angles are normalized, it is seen that for each face $\eta^{i}$ of $\sigma^{n}$ that has $\tau^{p}$ as a face, we have $\alpha\left(\bar{\eta}^{i}, P\right)=\alpha\left(\hat{\eta}^{i}, Q\right)$; hence $\alpha\left(\bar{\eta}^{i}, P\right)=\alpha\left(\eta^{i}, \sigma^{n}\right)$. Therefore, making use of the fact that $p$ is even, we see that in order to show (16), it suffices to show

$$
\sum_{i=1}^{n-p}(-1)^{i+1} \sum_{\bar{\eta}^{i}<P} \alpha\left(\bar{\eta}^{i}, P\right)=2 \alpha(0, P) .
$$

However, (19) is precisely the restatement for polyhedral cones of Sommerville's theorem concerning the angle sums and volume of convex spherical polytopes, and hence (19) is true. See p. 157 of [So] for the original statement of Sommerville's theorem, and see p. 174 of [Mc2] for the restatement of this theorem for polyhedral cones (note that $n-p$ is odd, which is needed to deduce (19) from Sommerville's theorem). 
We are now ready for the proof of Theorem 3.5.

Proof of Theorem 3.5. Let $\tau^{p}$ be a $p$-simplex of $K$. If $p=n-1$ or $p=n$, then $D_{n}^{a}\left(\tau^{p}\right)=0$ by Remark 3.3. Now assume that $0 \leq p \leq n-2$. If $p$ is odd, then $D_{n}^{a}\left(\tau^{p}\right)=0$ by Lemma 3.2(1) combined with the definition of $D_{n}^{a}\left(\tau^{p}\right)$. From now on assume that $p$ is even. We observe that the hypothesis on the links of even-dimensional simplices implies that every $(n-1)$-simplex of $K$ is the face of precisely two $n$-simplices. Hence we can apply Lemma 4.1 to $K$.

We compute

$$
\begin{aligned}
& \frac{1}{a_{p}} D_{n}^{a}\left(\tau^{p}\right)=\tilde{D}_{n}\left(\tau^{p}\right)+\frac{1}{2} \sum_{i=p+1}^{n-2}(-1)^{i-p} \sum_{\eta^{i} \succ \tau^{p}} \tilde{D}_{n}\left(\eta^{i}\right) \\
& =\left[T_{n}\left(\tau^{p}\right)-\sum_{\sigma^{n} \succ \tau^{p}} \alpha\left(\tau^{p}, \sigma^{n}\right)\right] \\
& +\frac{1}{2} \sum_{i=p+1}^{n-2}(-1)^{i} \sum_{\eta^{i} \succ \tau^{p}}\left[T_{n}\left(\eta^{i}\right)-\sum_{\sigma^{n} \succ \eta^{i}} \alpha\left(\eta^{i}, \sigma^{n}\right)\right] \\
& =1+\frac{1}{2} \sum_{i=p+1}^{n-2}(-1)^{i} \sum_{\eta^{i} \succ \tau^{p}} 1 \\
& -\sum_{\sigma^{n}>\tau^{p}} \alpha\left(\tau^{p}, \sigma^{n}\right)-\frac{1}{2} \sum_{i=p+1}^{n-2}(-1)^{i} \sum_{\eta^{i} \succ \tau^{p}} \sum_{\sigma^{n}>\eta^{i}} \alpha\left(\eta^{i}, \sigma^{n}\right) \\
& \text { (using Lemma 4.1(i), and doing some rearranging) } \\
& =1-\frac{1}{2} \sum_{k=0}^{n-p-3} \sum_{\omega^{k} \in \operatorname{link}\left(\tau^{p}, K\right)}(-1)^{k} \\
& -\sum_{\sigma^{n} \succ \tau^{p}} \alpha\left(\tau^{p}, \sigma^{n}\right)-\frac{1}{2} \sum_{\sigma^{n} \succ \tau^{p}} \sum_{i=p+1}^{n-2}(-1)^{i} \sum_{\sigma^{n} \succ \eta^{i} \succ \tau^{p}} \alpha\left(\eta^{i}, \sigma^{n}\right) \\
& =1-\frac{1}{2} \sum_{k=0}^{n-p-1} \sum_{\omega^{k} \in \operatorname{link}\left(\tau^{p}, K\right)}(-1)^{k}+\frac{1}{2} \sum_{\omega^{n-p-2} \in \operatorname{link}\left(\tau^{p}, K\right)}(-1)^{n-p-2} \\
& +\frac{1}{2} \sum_{\omega^{n-p-1} \in \operatorname{link}\left(\tau^{p}, K\right)}(-1)^{n-p-1} \\
& -\sum_{\sigma^{n} \succ \tau^{p}}\left\{\alpha\left(\tau^{p}, \sigma^{n}\right)-\frac{1}{2} \sum_{i=p+1}^{n-2}(-1)^{i+1} \sum_{\sigma^{n} \succ \eta^{i} \succ \tau^{p}} \alpha\left(\eta^{i}, \sigma^{n}\right)\right\} \\
& =1-\frac{1}{2} \chi\left(\operatorname{link}\left(\tau^{p}, K\right)\right)-\frac{1}{2} f_{n-p-2}\left(\operatorname{link}\left(\tau^{p}, K\right)\right) \\
& +\frac{1}{2} f_{n-p-1}\left(\operatorname{link}\left(\tau^{p}, K\right)\right)-\sum_{\sigma^{n} \succ \tau^{p}}\left\{\frac{1}{2}-\frac{n-p}{4}\right\}
\end{aligned}
$$

(using the fact that $n-p-2$ is odd, and (15)) 


$$
\begin{aligned}
= & 1-\frac{1}{2} \cdot 2-\frac{1}{2} \frac{n-p}{2} f_{n-p-1}\left(\operatorname{link}\left(\tau^{p}, K\right)\right) \\
& +\frac{1}{2} f_{n-p-1}\left(\operatorname{link}\left(\tau^{p}, K\right)\right)-f_{n-p-1}\left(\operatorname{link}\left(\tau^{p}, K\right)\right)\left(\frac{1}{2}-\frac{n-p}{4}\right)
\end{aligned}
$$

(using the hypothesis of the theorem, and Lemma 4.1(ii))

$$
=0 \text {, }
$$

where the equality before the last uses the fact that $f_{n-p-1}\left(\operatorname{link}\left(\tau^{p}, K\right)\right)$ equals the number of $n$-simplices of $K$ that have $\tau^{p}$ as a face.

Finally, we have the proof of Theorem 3.9.

Proof of Theorem 3.9. To prove (9), we first observe that if either $s$ or $p$ is odd, then it follows from Remark 3.3(2) that both sides of (9) are zero, and so the equation holds. From now on, assume that both $s$ and $p$ are even. Clearly $s \leq p$.

We start with the following preliminary. Suppose that $\alpha^{r}$ is an $r$-simplex of $K$ that has $\zeta^{p}$ as a face, where $p+1 \leq r \leq n-2$. We then claim that

$$
\sum_{i=s+1}^{r} \sum_{\substack{\eta^{i} \in L \\ \eta^{i} \leq \alpha^{r} \\ \eta^{i}>\tau^{s}}}(-1)^{i-s}=(-1)^{r-s} .
$$

To see why (20) holds, we first observe that

$$
\bigcup_{\substack{\eta^{i} \in L \\ \eta^{i} \leq \alpha^{r} \\ \eta^{i}>\tau^{s}}} \eta^{i}=\left|\operatorname{star}\left(\tau^{s}, L\right)\right| \cap \alpha^{r}
$$

where, as always, we are thinking of simplices as open. Let $T=\left\{\omega^{k} \in \operatorname{link}\left(\tau^{s}, L\right) \mid\right.$ $\left.\omega^{k} \subseteq \alpha^{r}\right\}$. Observe that $|T|$ is an open $(r-s-1)$-disk. We now have

$$
\sum_{i=s+1}^{r} \sum_{\substack{\eta^{i} \in L \\ \eta^{i} \leq \alpha^{r} \\ \eta^{i}>\tau^{s}}}(-1)^{i-s-1}=\sum_{k=0}^{r-s-1} \sum_{\omega^{k} \in T}(-1)^{k}=\sum_{k=0}^{r-s-1}(-1)^{k} f_{k}(T) .
$$

We note that the sum $\sum_{k=0}^{r-s-1}(-1)^{k} f_{k}(T)$ is not necessarily equal to $\chi(T)$, because $T$ is not a simplicial complex. However, we note that $\bar{T}$, the closure of $T$, is a simplicial complex (in particular, it is an $(r-s-1)$-disk). Hence, using the discussion in Section 2 of [Bl1], it is seen that

$$
\sum_{k=0}^{r-s-1}(-1)^{k} f_{k}(T)=(-1)^{r-s-1} .
$$

If we combine (22) and (23), and multiply through by -1 , we deduce (20).

One more preliminary observation. Suppose that $v^{i}$ is an $i$-simplex of $L$ that is contained in a (unique) $r$-simplex $\mu^{r}$ of $K$. We observe that $T_{n}\left(v^{i}\right)=T_{n}\left(\mu^{r}\right)$ and 
$\tilde{D}_{n}\left(v^{i}\right)=\tilde{D}_{n}\left(\mu^{r}\right)$, where the left-hand side of each of these equations is with respect to $L$, and the right-hand side is with respect to $K$ (in general, if we write " $\tilde{D}_{n}\left(\eta^{i}\right)$," that is with respect to the simplicial complex of which $\eta^{i}$ is a simplex, which is always clear from context).

We can now demonstrate (9), by computing

$$
\begin{aligned}
& a_{p} D_{n}^{a}\left(\tau^{s}\right)=a_{p} a_{s} \tilde{D}_{n}\left(\tau^{s}\right)+a_{p} \frac{a_{s}}{2} \sum_{i=s+1}^{n-2}(-1)^{i-s} \sum_{\substack{\eta^{i} \in L \\
\eta^{i}>\tau^{s}}} \tilde{D}_{n}\left(\eta^{i}\right) \\
& =a_{p} a_{s} \tilde{D}_{n}\left(\zeta^{p}\right)+\frac{a_{p} a_{s}}{2} \sum_{\substack{\eta^{i} \in L \\
s+1 \leq i \leq n-2 \\
\eta^{i}>\tau^{s}}}(-1)^{i-s} \tilde{D}_{n}\left(\eta^{i}\right) \\
& =a_{p} a_{s} \tilde{D}_{n}\left(\zeta^{p}\right)+\frac{a_{p} a_{s}}{2} \sum_{\substack{\alpha^{r} \in K \\
p+1 \leq r \leq n \\
\alpha^{\prime}>\zeta^{p}}} \sum_{\substack{\eta^{i} \in L \\
\eta^{i} \in \alpha^{r} \\
s+1 \leq i \leq \min _{i}\{, n-2\} \\
\eta^{i}>\tau^{s}}}(-1)^{i-s} \tilde{D}_{n}\left(\alpha^{r}\right) \\
& =a_{p} a_{s} \tilde{D}_{n}\left(\zeta^{p}\right)+\frac{a_{p} a_{s}}{2} \sum_{r=p+1}^{n} \sum_{\substack{\alpha^{r} \in K \\
\alpha^{r} \succ \zeta^{p}}} \tilde{D}_{n}\left(\alpha^{r}\right) \sum_{\substack { i=s+1 \\
\begin{subarray}{c}{n^{i} \in L \\
n^{i} \leq \alpha^{r} \\
\eta^{\prime} \succ \tau^{s}{ i = s + 1 \\
\begin{subarray} { c } { n ^ { i } \in L \\
n ^ { i } \leq \alpha ^ { r } \\
\eta ^ { \prime } \succ \tau ^ { s } } }\end{subarray}}(-1)^{i-s} \\
& \text { (because } \tilde{D}_{n}\left(\alpha^{r}\right)=0 \text { for } r=n-1 \text { or } r=n \text { ) } \\
& =a_{s} a_{p} \tilde{D}_{n}\left(\zeta^{p}\right)+a_{s} \frac{a_{p}}{2} \sum_{r=p+1}^{n-2} \sum_{\substack{\alpha^{r} \in K \\
\alpha^{r} \succ \succ^{p}}} \tilde{D}_{n}\left(\alpha^{r}\right)(-1)^{r-p} \\
& \text { (using (20), and the fact that both } s \text { and } p \text { are even) } \\
& =a_{s} D_{n}^{a}\left(\zeta^{p}\right) .
\end{aligned}
$$

Finally, to deduce (10), assume $s=p$. There are two cases to consider. When $a_{s}=a_{p}=0$, then both sides of (10) are zero by definition. When $a_{s}=a_{p} \neq 0$, then (10) follows from (9).

\section{Acknowledgments}

The author thanks Tom Banchoff, Mark Halsey, Walter Neumann, Guyan Robertson, Lauren Rose, Rebecca Thomas, and Min Yan for many helpful suggestions.

\section{References}

[AS] M. Abramowitz and I. Stegun, Handbook of Mathematical Functions with Formulas, Graphs, and Mathematical Tables, Applied Mathematics Series, vol. 55, National Bureau of Standards, Washington, DC, 1972.

[Ba1] T. Banchoff, Critical points and curvature for embedded polyhedra, J. Diff. Geom. 1 (1967), 245-256.

[Ba2] T. Banchoff, Critical points and curvature for embedded polyhedral surfaces, Amer. Math. Monthly 77 (1970), 475-485.

[Ba3] T. Banchoff, Critical points and curvature for embedded polyhedra, II, Progress in Math. 32 (1983), $34-55$. 
[B11] E. D. Bloch, The angle defect for arbitrary polyhedra, Beiträge Algebra Geom. 39 (1998), 379-393.

[B12] E. D. Bloch, Critical points and the angle defect, Geom. Dedicata 109 (2004), 121-137.

[Bu] L. Budach, Lipschitz-Killing curvatures of angular partially ordered sets, Adv. Math. 78 (1989), 140167.

[C] J. Cheeger, Spectral geometry of singular Riemannian spaces, J. Differential Geom. 18 (1983), 575657.

[Ch1] B. Chen, The Gram-Sommerville and Gauss-Bonnet theorems and combinatorial geometric measures for noncompact polyhedra, Adv. Math. 91 (1992), 269-291.

[Ch2] B. Chen, Weight functions, double reciprocity laws, and volume formulas for lattice polyhedra, Proc. Natl. Acad. Sci. USA 95 (1998), 9093-9098.

[CMS] J. Cheeger, W. Muller, and R. Schrader, On the curvature of piecewise flat spaces, Comm. Math. Phys. 92 (1984), 405-454.

[CY1] B. Chen and M. Yan, Eulerian stratification of polyhedra, Adv. in Appl. Math. 21 (1998), 22-57.

[CY2] B. Chen and M. Yan, Eulerian 2-strata spaces, J. Combin. Theory Ser. A 85 (1999), 1-28.

[F] P. J. Federico, Descartes on Polyhedra, Springer-Verlag, New York, 1982.

[GKP] R. Graham, D. Knuth, and O. Patashnik, Concrete Mathematics, 2nd edn., Addison-Wesley, Reading, MA, 1994.

[Gr1] B. Grünbaum, Convex Polytopes, Wiley, New York, 1967.

[Gr2] B. Grünbaum, Grassmann angles of convex polytopes, Acta. Math. 121 (1968), 293-302.

[GS] B. Grünbaum and G. C. Shephard, Descartes' theorem in $n$ dimensions, Enseign. Math. (2) 37 (1991), $11-15$.

[Gu] A. P. Guinand, A note on the angles in an $n$-dimensional simplex, Proc. Glasgow Math. Assoc. 4 (1959), 58-61.

[H] J. F. P. Hudson, Piecewise Linear Topology, Benjamin, Menlo Park, CA, 1969.

[HT] S. Halperin and D. Toledo, Stiefel-Whitney homology classes, Ann. of Math. (2) 96 (1972), 511-525.

[Kl] V. Klee, A combinatorial analogue of Poincaré's duality theorem, Canad.J. Math. 16 (1964), 517-531.

[Kn] K. Knopp, Theory and Application of Infinite Series, Blackie, London, 1928.

[MR] C. MacLaurin and G. Robertson, Euler characteristic in odd dimensions, Austral. Math. Soc. Gaz. 30 (2003), 195-199.

[Mc1] P. McMullen, Non-linear angle-sum relations for polyhedral cones and polytopes, Math. Proc. Cambridge Philos. Soc. 78 (1975), 247-261.

[Mc2] P. McMullen, Angle-sum relations for polyhedral sets, Mathematika 33 (1986), 173-188.

[MS] J. W. Milnor and J. Stasheff, Characteristic Classes, Annals of Mathematics Studies, vol. 76, Princeton University Press, Princeton, NJ, 1974.

[Mu] J. R. Munkres, Elements of Algebraic Topology, Addison-Wesley, Menlo Park, CA, 1984.

[P] E. Peschl, Winkelrelationen am Simplex und die Eulersche Charakteristik, Bayer. Akad. Wiss. Math.Natur. Kl. Sitzungsber 1955 (1956), 319-345.

[PS] M. A. Perles and G. C. Shephard, Angle sums of convex polytopes, Math. Scand. 21 (1967), 199-218.

[RW] M. Roček and R. M. Williams, On the Euler characteristic for piecewise linear manifolds, Phys. Lett. B 273 (1991), 95-99.

[Sh1] G. C. Shephard, An elementary proof of Gram's theorem for convex polytopes, Canad. J. Math. 19 (1967), 1214-1217.

[Sh2] G. C. Shephard, Angle deficiencies of convex polytopes, J. London Math. Soc. 43 (1968), 325-336.

[S1] N. J. A. Sloane, The On-Line Encyclopedia of Integer Sequences, http://www.research.att.com/ njas/sequences.

[So] D. M. Y. Sommerville, An Introduction to the Geometry of n Dimensions, Methuen, London, 1929.

[Su] D. Sullivan, Combinatorial invariants of analytic spaces, Proc. Liverpool Singularities Symposium I (1969/70), Lecture Notes in Mathematics, vol. 192, Springer-Verlag, Berlin, 1971, pp. 165-168.

[W] P. Wintgen, Normal cycle and integral curvature for polyhedra in Riemannian manifolds, in Differential Geometry (Gy. Soós and J. Szenthe, eds.), Colloquia Mathematica Societati János Bolyai, vol. 31, North-Holland, Amsterdam, 1982, pp. 805-816.

[Z] M. Zähle, Approximation and characterization of generalized Lipschitz-Killing curvatures, Ann. Global Annal. Geom. 8 (1990), 249-260.

Received December 4, 2003, and in revised form August 14, 2005. Online publication December 30, 2005. 